\title{
Soliton-assisted random lasing in optically-pumped liquid crystals
}

\author{
Sreekanth Perumbilavil, ${ }^{1}$ Armando Piccardi, ${ }^{2}$ Oleksandr Buchnev, ${ }^{3}$ \\ Martti Kauranen, ${ }^{1}$ Giuseppe Strangi ${ }^{4,5}$ and Gaetano Assanto ${ }^{1,2,6, *}$ \\ ${ }^{1}$ Optics Laboratory, Tampere University of Technology, FI-33101 Tampere, Finland \\ ${ }^{2}$ NooEL - Nonlinear Optics and OptoElectronics Lab, University "Roma Tre", IT-00146 Rome, Italy \\ ${ }^{3}$ Optoelectronics Research Centre, University of Southampton, SO17 1BJ, Southampton, United Kingdom \\ ${ }^{4}$ Dept. of Physics, Case Western Reserve University, Cleveland, Ohio 44106-7079, USA \\ ${ }^{5}$ CNR Nanotec, Rende and Italian Institute of Technology, Genova, Italy \\ ${ }^{6}$ CNR-ISC, Institute for Complex Systems, IT-00185 Rome, Italy
}

(Dated: September 22, 2016)

\begin{abstract}
We demonstrate a novel random laser configuration by exploiting the coexistence of optical gain and light self-localization in a reorientational nonlinear medium. A spatial soliton launched by a near-infrared beam in dye-doped nematic liquid crystals enhances and confines stimulated emission of visible light in the optically-pumped gain-medium, yielding random lasing with enhanced features.
\end{abstract}

In random lasers, a disordered distribution of scattering centers provides the required feedback for oscillations in optically amplifying media. In recent years they have attracted a great deal of attention, mainly due to the versatility stemming from cavity-less geometries and the ease of realization [1-9]. In liquid crystals, suitable dopants can provide the gain action through optical pumping, while optical birefringence in conjunction with intense fluctuations of the dielectric tensor yield the required recurrent multiple scattering for random resonances to occur [10-17]. In the nematic phase, moreover, liquid crystals are positive uniaxial materials subject to optic axis reorientation under the action of electric fields, either at low or optical frequencies [18]. The latter response provides a low-power mechanism for nonlinear optics [19] and light localization into self-confined lightbeams, the so-called "nematicons" [20]. Nematicons are bright spatial solitons (solitary waves) which are stable in two transverse dimensions due to the nonlocal response associated with the elastic intermolecular links in the liquid state [21, 22]; they support graded-index waveguides able to confine additional (incoherent) signals/beams of different wavelengths as well as powers and profiles [2330], are robust against refractive index perturbations [3135] and collisional interactions [36-38]. Aided by nematicons, reorientational and electronic nonlinear responses, characterized by distinct time- and power-scales, can synergystically be combined $[39,40]$. Owing to their large numerical aperture [41], nematicon waveguides solitons have also been employed in experiments involving incoherent light generation by fluorescence [42] or amplified spontaneous emission [43], offering a means to better collect and couple the emitted light into optical fibers.

In this Letter we demonstrate a novel example of synergy between diverse nonlinear responses: the combination of spatial solitons and random lasing into a "nematicon random laser", whereby a low-power self-confined beam provides a guided-wave landscape for the stimu-

*assanto@uniroma3.it lated emission induced by collinear optical pumping of dye-doped nematic liquid crystals (NLC).

Several benefits can be expected from adopting such light-induced guided-wave configuration for random lasing. At variance with standard thin film geometries, the thick NLC cell provides an extended volume where optical pumping can produce fluorescence and, in turn, stimulated emission and lasing action via random scattering and feedback. The large interaction volume can exploit several feedback paths through scattering, support many coupled lasing modes [44] and their competition/thermalization [45], producing smoother spectrum and spatial profile as compared to "standard" random lasers. Furthermore, owing to anisotropic light scattering in uniaxials [46], the spontaneous as well as the stimulated emissions in NLC doped with pyrromethene-dye tend to be polarized in the plane of the director alignment $[10,43]$; hence, the generated photons are co-polarized with the reorientational soliton and can be trapped in the light-induced waveguide, the nematicon. At variance with a standard one-beam configuration (see Fig. $1(\mathrm{a}))$, the confinement afforded by the nematicon on extraordinary-polarized (e-) waves can balance out the spatial dispersion of the involved wavepackets and result in reduced diffraction through index change as well as modified distribution of the emission through disorder confinement [47] and altered scattering. In standard NLC random lasers, localized and extended modes in the active volume overlap and compete; in our geometry transversely localized modes are sustained by the waveguide landscape and conveyed at the exit. Finally, the pump beam can also undergo nematicon confinement depending on its polarization: if polarized along $y$ at the input, even the e-pump can be guided along the nematicon, yielding an extended interaction with light emitted along the waveguide and only limited by absorption. The latter pump polarization, however, turns out to be the least efficient in terms of fluorescence in the uniaxial guesthost material. For this reason, we conducted experiments with either (i) an ordinary-wave (o-) pump with polarization orthogonal to the optic axis or (ii) an extraordinary 
pump copolarized with (and guided by) the soliton and the emitted light (Fig. 1(b-c).

We prepared and employed planar glass cells with mechanically rubbed polymide/NLC interfaces to prepare $100 \mu \mathrm{m}$ thick samples of nematic liquid crystals E7 doped with $0.3 \mathrm{wt} \%$ pyrromethene dye (PM597). Such guesthost mixture, when resonantly pumped by nanosecond laser pulses at $532 \mathrm{~nm}$, is able to provide fluorescence and optical amplification in the visible spectrum around $570-580 \mathrm{~nm}[10,43]$. When excited by $\mathrm{mW}$-power e-wave beams, through non-resonant reorientation it can also support the formation and propagation of self-guided spatial optical solitons of arbitrary wavelength [20]. The NLC cell was $3 \mathrm{~mm}$-long in propagation and equipped with input and output glass interfaces to prevent meniscus formation and undesired beam depolarization (see sample geometry in Fig. 1(d)). The NLC optic axis was aligned in the plane $y z$ at $\theta=45^{\circ}$ with respect to the wave vector direction $\mathbf{k} / / z$ in order to avoid a power (Freedericks) threshold effect for all-optical reorientation while maximizing the nonlinear optical response [48].

A cw near-infrared beam of wavelength $1.064 \mu \mathrm{m}$ was e-polarized with electric field parallel to $y$, focused at the input interface to a waist $3 \mu \mathrm{m}$ and launched with wave vector along $z$ in the cell mid-plane $(x=0)$ in order to excite a self-trapped nematicon (see Fig.1(e)). The latter, stabilized by nonlocality in two-dimensions, is able to propagate with a nearly invariant profile in the plane $y z$ at the walkoff angle $\delta$ defined by the birefringence $[20,21]$, i.e., $\delta=\arctan \left[\frac{\left(n_{\|}^{2}-n_{\perp}^{2}\right) \sin \theta \cos \theta}{n_{\|}^{2} \sin ^{2} \theta+n_{\perp}^{2} \cos ^{2} \theta}\right]$, with $n_{\|}$and $n_{\perp}$ the refractive indices for electric fields parallel and orthogonal to the optic axis $\hat{\mathbf{n}}$, respectively. A $20 \mathrm{~Hz}$ reprate train of $\approx 6$ ns pulses from a frequency-doubled Qswitched Nd:YAG laser was focused and injected in the cell mid-plane collinearly with the $\mathrm{cw}$ beam, with particular care to ensure that, despite walkoff, the two (green and near-infrared) beams had parallel Poynting vectors. The experimental setup was completed by a microscope, a CCD camera to check the beam alignment and a spectrometer to collect the emission from the cell output, as sketched in Fig. 1(e).

Figure 2(a) shows the measured output versus input characteristics of the random laser, acquired from the emitted light without (black) and with (red) a $5 \mathrm{~mW}$ nematicon, injecting the pump in the e-polarization. The emission, collected after a bandpass filter and spatially integrated, exhibits the standard lasing character with an energy threshold around $0.85 \mu \mathrm{J}$ and a higher slope (efficiency) when the nematicon is present. Fig. 2(b) displays the corresponding results for an ordinary-polarized pump: as before, the nematicon increases the lasing efficiency. For o-polarized pump light the threshold is slightly lower and a marked increase in the output-vsinput slope and lasing efficiency is clearly visible, consistently with previous observations in the same material $[10,43]$ In the latter geometry, the o-pump is not confined but interacts more efficiently with the guest-host, (a)

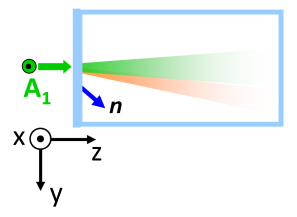

(d)
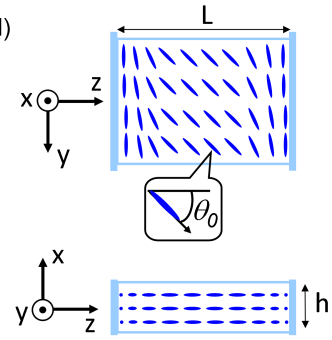

(c)
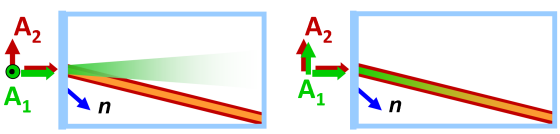

(e)

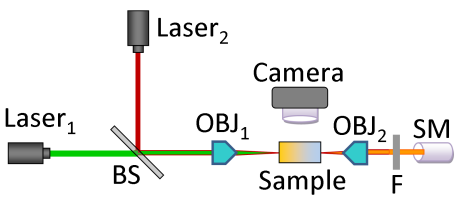

FIG. 1. (a) Sketch of the interaction without nematicon. (b-c) Sketch of the interaction with nematicon (amplitude $A_{2}$ ) and (b) ordinary or (b) extraordinary wave pump (amplitude $A_{1}$ ). The emitted light, represented here as a beam, is actually generated in the region where the pump remains intense despite the absorption.(d) Sample geometry and director alignment. $\theta_{0}$ is the bulk orientation due to the boundary conditions. (e) Experimental setup: SM, spectrometer; OBJ, microscope objective; Laser 1 , pulsed pump laser at 532nm; Laser 2 , cw laser at $1064 \mathrm{~nm}$.

whereas the e-wave emission is confined within the nearinfrared nematicon and propagates along its walkoff angle (Fig. 1(b)). Clearly, despite the spatial spreading incurred by the o-polarized pump, the random laser operates better in this configuration with orthogonal pump and soliton. When calculating the $\beta$-factor (rate of spontaneous emission radiated into lasing modes over total rate of spontaneous emission) according to the procedure outlined in Ref. [49] and used, e.g., in Ref. [50], the $\beta$ reduction observed for an o-wave pump became more apparent in the presence of the nematicon, as indicated by the values next to each curve in Fig. 2. This confirms that the fraction of spontaneous emission that triggers random lasing is significantly less with than without soliton, i.e., the nematicon-assisted configuration is more efficient.

Figure 3 illustrates the spectral narrowing afforded by this random laser with/without a collinear spatial soliton. The full-width half-maximum data points, plotted versus pump energy/pulse, clearly show the lasing characteristics corresponding to Fig. 2, with a marked improvement when the nematicon contributes to the confinement of the emitted light. A narrowing of the emitted spectrum is visible in (a) without soliton as the system goes from fluorescence to above lasing threshold, from $43.4 \mathrm{~nm}(45.6 \mathrm{~nm})$ to $11.0 \mathrm{~nm}(19.8 \mathrm{~nm})$ for o- (e-) pump; the spectral narrowing is more marked with nematicon assistance, particularly for a pump orthogonal to the optic axis of the guest-host medium: from $42.9 \mathrm{~nm}(43.8 \mathrm{~nm})$ to $7.2 \mathrm{~nm}(12.3 \mathrm{~nm})$ for o- (e-) pump. The spectral width 

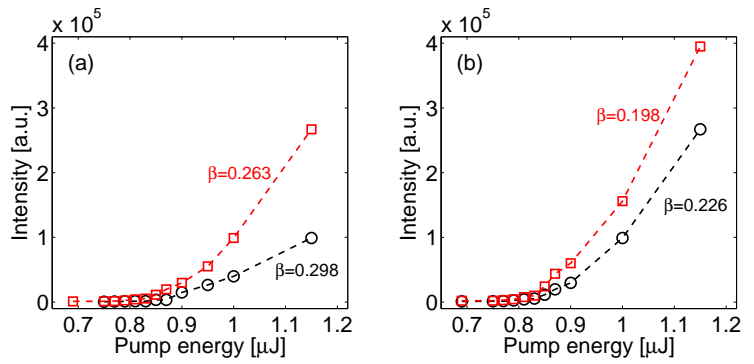

FIG. 2. Output intensity of emitted light (units correspond to spectrometer counts) at wavelengths around $575 \mathrm{~nm}$ the energy of the pump pulse. (a) e-wave pump in bulk interaction without nematicon (black symbols and line) and guided-wave interaction in the presence of a $5 \mathrm{~mW}$ nematicon (red symbols and line); (b) o-wave pump without (black symbols and line) and with a $5 \mathrm{~mW}$ nematicon (red symbols and line). The calculated $\beta$-factors are indicated next to each line.
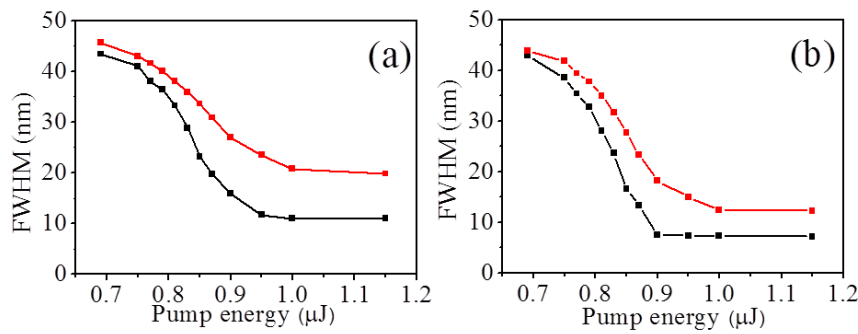

FIG. 3. Measured full-width half-maxima (FWHM) of the spectral emission (see Fig. 4) versus energy of the pump pulses, for an ordinary (black symbols and lines) or extraordinary (red symbols and lines) wave pump (a) in a bulk interaction (no soliton), (b) in the presence of a collinear $5 \mathrm{~mW}$ nematicon.

in the presence of a nematicon is consistently lower above threshold, confirming the beneficial role of our solitin assisted geometry in an extended interaction region.

Figure 4 shows various lasing spectra when the guesthost system is pumped (o-polarization) in the presence/absence of a collinear nematicon. When the pump energy is below threshold $(0.75 \mu \mathrm{J})$ no laser action takes place and a $5 \mathrm{~mW}$ nematicon barely increases the emitted intensity by collecting photons at the output (Fig. $4(\mathrm{a}))$. Conversely, pumping above threshold $(1.2 \mu \mathrm{J})$, the index contrast introduced by the nematicon through reorientation assists the emission process by changing the scattering paths and the interaction efficiency, resulting in wavelength tuning and spectral narrowing (Fig. 4(b)). Fig. 4(c-d) illustrates the nematicon role on spectral profiles, width and random laser intensity for $1.2 \mu \mathrm{J}$ o-wave pumping and nematicon power varying up to $6 \mathrm{~mW}$. Such all-optical tuning can be ascribed to changes in the local scattering cross-section that, in turn, modify the transport distance of the generated photons and the size of the random loops responsible for random resonances and lasing.

Finally, Fig. 5 graphs the spatial profile of the emit-
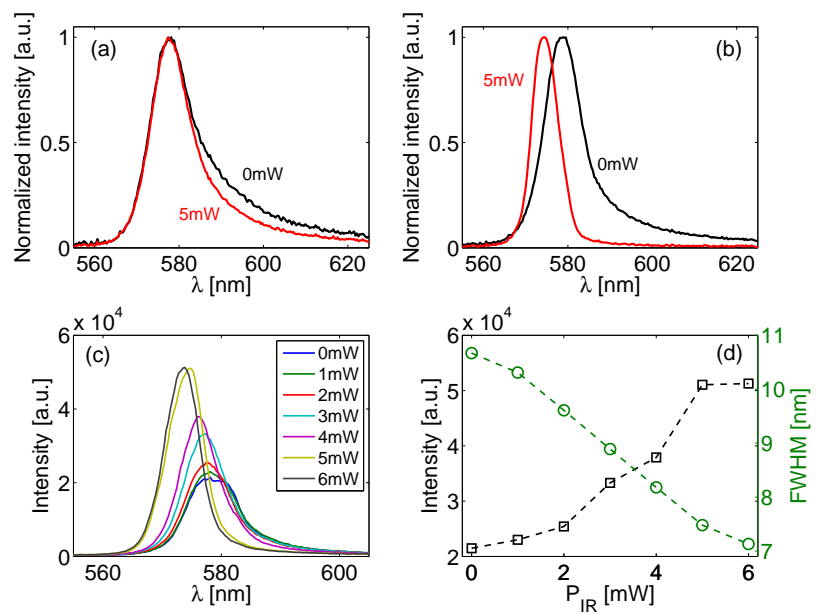

FIG. 4. Soliton effects on spectral emission, for a pump orthogonal to the optic axis. (a) Emission spectrum with pump energy below threshold $(0.75 \mu \mathrm{J})$, without (black) and with a $5 \mathrm{~mW}$ soliton (red). (b) Spectrum with pump energy above threshold $(1.2 \mu \mathrm{J})$, without (black) and with (red) a $5 \mathrm{~mW}$ nematicon: the latter shifts the wavelength peak and narrows the spectrum. (c) Emission spectra for a pump at $1.2 \mu \mathrm{J}$ and various nematicon powers. (d) Tuning of lasing efficiency (output intensity, squares) and spectral width (FWHM, circles) versus nematicon power, for a pump at $1.2 \mu \mathrm{J}$.

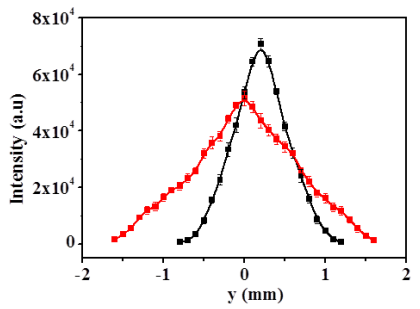

FIG. 5. Measured spatial distribution of the random laser emission, imaged with a microscope objective by translating a slit and filtering out near-infrared and green light, with (black symbols and line) and without (red symbols and line) a collinear $5 \mathrm{~mW}$ nematicon. The peak emission is at the wavelength $578.1 \mathrm{~nm}$ without soliton and at $573.9 \mathrm{~nm}$ with soliton, respectively.

ted light.The curves, plotted against the transverse coordinate $y$, indicate that the soliton confines the laser light and improves its transverse distribution and spatial coherence, consistently with the expected benefits of a nematicon randon laser. When exploited in conjunction with nematicon steering and readdressing, these features can allow one to engineer random lasing with increased spatial coherence and controlled direction of emission.

In conclusion, we have designed and demonstrated the first nematicon-assisted random laser, where the graded index region defined by the spatial soliton through reorientation confines the emitted radiation and contributes to tune the features of random lasing in terms of spec- 
trum and overall efficiency, as witnessed by changes in $\beta$ factor, laser wavelength and linewidth. While these preliminary results are sensitive to details of sample preparation and definitely subject to improvement through a better control of beam alignment and guest-host composition, they prove that the benefits of a cavity-less random laser can be combined with those of an optical control of scattering and diffraction by a light-induced guided-wave geometry, towards better laser coherence and directionality, narrower lines and lower threshold with higher slope efficiency. We plan to investigate additional properties of nematicon random lasers and to derive a suitable model accounting for the large number of lasing modes and their competition in an extended and confined interaction region.

\section{ACKNOWLEDGMENTS}

This work was supported by the Academy of Finland through the Finland Distinguished Professor grant no. 282858. The authors also thank C. Conti for enlightening discussions and the European Union COST Action IC1208.
[1] D. S. Wiersma and S. Cavalieri, Nature 414, 708 (2001).

[2] D. S. Wiersma, Nature Phys. 4, 359 (2008).

[3] M. Leonetti, C. Conti, and C. Lopez, Nat. Photon. 5, 615 (2011).

[4] N. Bachelard, J. Andreasen, S. Gigan, and P. Sebbah, Phys. Rev. Lett. 109, 033903 (2012).

[5] Y. Bliokh, E. I. Chaikina, N. Lizárraga, E. R. Méndez, V. Freilikher, and F. Nori, Phys. Rev. B 86, 054204 (2012).

[6] M. Leonetti and C. Lopez, Appl. Phys. Lett. 102, 071105 (2013).

[7] S. Turitsyn, S. A. Babin, D. Churkin, I. D. Vatnik, M. Nikulin, and E. Podivilov, Phys. Rep. 542, 133 (2014).

[8] C. Dominques, M. A. Gomes, Z. Macedo, C. B. de Araujo, and A. S. L. Gomes, Nanoscale 7, 317 (2015).

[9] L. V. Gerasimov, D. V. Kuprianov, and M.D.Havey, Opt. Spectr. 119, 377 (2015).

[10] G. Strangi, S. Ferjani, V. Barna, A. D. Luca, C. Versace, N. Scaramuzza, and R. Bartolino, Opt. Express 14, 7737 (2006).

[11] S. Ferjani, L. Sorriso-Valvo, A. De Luca, V. Barna, R. De Marco, and G. Strangi, Phys. Rev. E 78, 011707 (2008).

[12] B. He, Q. Liao, and Y. Huang, Opt. Mat. 31, 375 (2008).

[13] Q. Song, L. Liu, L. Xu, Y. Wu, and Z. Wang, Opt. Lett. 34, 298 (2009).

[14] C.-R. Lee, J.-D. Lin, B.-Y. Huang, T.-S. Mo, and S.-Y. Huang, Opt. Express 18, 25896 (2010).

[15] C.-R. Lee, J.-D. Lin, B.-Y. Huang, S.-H. Lin, T.-S. Mo, S.-Y. Huang, C.-T. Kuo, and H.-C. Yeh, Opt. Express 19, 2391 (2011)

[16] S. M. Morris, D. J.Gardiner, M. M. Qasim, P. J. W.Hands, T. D. Wilkinson, and H. J. Coles, J. Appl. Phys. 111, 033106 (2012).

[17] H. Biana, F. Yaoa, H. Liuc, F. Huanga, Y. Peiab, C. Houab, and X. Sunab, Liq. Cryst. 41, 1436 (2014).

[18] P. G. DeGennes and J. Prost, The Physics of Liquid Crystals (Oxford Science, New York, 1993).

[19] I. C. Khoo, Phys. Rep. 471, 221 (2009).

[20] M. Peccianti and G. Assanto, Phys. Rep. 516, 147 (2012).

[21] C. Conti, M. Peccianti, and G. Assanto, Phys. Rev. Lett. 92, 113902 (2004).

[22] A. Alberucci, C. P. Jisha, N. Smyth, and G. Assanto, Phys. Rev. A 91, 013841 (2015).
[23] M. Peccianti and G. Assanto, Opt. Lett. 26, 1791 (2001).

[24] X. Hutsebaut, C. Cambournac, M. Haelterman, J. Beeckman, and K. Neyts, J. Opt. Soc. Am. B 22, 1424 (2005).

[25] A. Alberucci, M. Peccianti, G. Assanto, A. Dyadyusha, and M. Kaczmarek, Phys. Rev. Lett. 97, 153903 (2006).

[26] J. F. Henninot, J. F. Blach, and M. Warenghem, J. Opt. A- Pure Appl. Opt. 9, 20 (2007).

[27] Y. Izdebskaya, V. Shvedov, A. Desyatnikov, W. Krolikowski, M. Belic, G. Assanto, and Y. Kivshar, Opt. Express 18, 3258 (2010).

[28] A. Piccardi, A. Alberucci, and G. Assanto, Phys. Rev. Lett. 104, 213904 (2010).

[29] Y. Izdebskaya, A. Desyatnikov, G. Assanto, and Y. Kivshar, Opt. Lett. 36, 184 (2011).

[30] Y. Izdebskaya, G. Assanto, and W. Krolikowski, Opt. Lett. 40, 4182 (2015).

[31] A. Pasquazi, A. Alberucci, M. Peccianti, and G. Assanto, Appl. Phys. Lett. 87, 261104 (2005).

[32] A. Piccardi, G. Assanto, L. Lucchetti, and F. Simoni, Appl. Phys. Lett. 93, 171104 (2008).

[33] A. Piccardi, A. Alberucci, U. Bortolozzo, S. Residori, and G. Assanto, IEEE Photon. Techn. Lett. 22, 694 (2010).

[34] Y. Izdebskaya, V. Shvedov, A. Desyatnikov, W. Krolikowski, and Y. Kivshar, Opt. Lett. 35, 1692 (2010).

[35] A. Piccardi, A. Alberucci, U. Bortolozzo, S. Residori, and G. Assanto, Appl. Phys. Lett. 96, 071104 (2010).

[36] M. Peccianti, K. Brzadkiewicz, and G. Assanto, Opt. Lett. 27, 1460 (2002).

[37] C. P. Jisha, A. Alberucci, R.-K. Lee, and G. Assanto, Opt. Lett. 36, 1848 (2011).

[38] A. Piccardi, S. Residori, and G. Assanto, J. Opt. 18, 07LT01 (2016).

[39] I. Burgess, M. Peccianti, G. Assanto, and R. Morandotti, Phys. Rev. Lett. 102 (2009).

[40] M. Peccianti, A. Pasquazi, G. Assanto, and R. Morandotti, Opt. Lett. 35, 3342 (2010).

[41] M. Peccianti and G. Assanto, Opt. Lett. 26, 1690 (2001).

[42] J. F. Henninot, J. F. Blach, and M. Warenghem, J. Appl. Phys. 107 (2010)

[43] S. Bolis, T. Virgili, S. K. Rajendran, J. Beeckman, and P. Kockaert, Opt. Lett. 41, 2245 (2016).

[44] M. Leonetti, C. Conti, and C. Lopez, Light Sci. Appl. 2, e88 (2013). 
[45] C. Conti, M. Leonetti, A. Fratalocchi, L. Angelani, and G. Ruocco, Phys. Rev. Lett. 101 (2008).

[46] S. Gottardo, S. Cavalieri, O. Yaroshchuk, and D. S. Wiersma, Phys. Rev. Lett. 93, 263901 (2004).

[47] N. Ghofraniha, I. Viola, A. Zacheo, V. Arima, G. Gigli, and C. Conti, Opt. Lett. 38, 5043 (2013).
[48] M. Peccianti, C. Conti, and G. Assanto, Opt. Lett. 30, 415 (2005).

[49] G. van Soest and A. Lagendijk, Phys. Rev. E 65, 047602 (2002).

[50] S. Ferjani, A. D. Luca, V. Barna, C. Versace, and G. Strangi, Opt. Express 17, 2042 (2009). 